\title{
Multi-level mining and visualization of scientific text collections
}

\author{
Exploring a bilingual scientific repository \\ Pablo Accuosto, Francesco Ronzano, Daniel Ferrés, Horacio Saggion \\ Large-Scale Text Understanding Systems Lab \\ TALN Research Group \\ Department of Information and Communication Technologies \\ Universitat Pompeu Fabra \\ $\mathrm{C} /$ Tanger 122 \\ Barcelona, Spain 08018 \\ \{name.surname\}@upf.edu
}

\begin{abstract}
We present a system to mine and visualize collections of scientific documents by semantically browsing information extracted from single publications or aggregated throughout corpora of articles. The text mining tool performs deep analysis of document collections allowing the extraction and interpretation of research paper's contents. In addition to the extraction and enrichment of documents with metadata (titles, authors, affiliations, etc), the deep analysis performed comprises semantic interpretation, rhetorical analysis of sentences, triple-based information extraction, and text summarization. The visualization components allow geographicalbased exploration of collections, topic-evolution interpretation, and collaborative network analysis among others. The paper presents a case study of a bilingual collection in the field of Natural Language Processing (NLP).
\end{abstract}

\section{KEYWORDS}

Language Resources, Scientific Text Mining, Information Extraction, Data Visualization, PDF Conversion, Big Scientific Data

\section{ACM Reference format:}

Pablo Accuosto, Francesco Ronzano, Daniel Ferrés, Horacio Saggion. 2017. Multi-level mining and visualization of scientific text collections. In Proceedings of The 6st International Workshop on Mining Scientific Publications. Joint Conference on Digital Libraries, Toronto, Canada, June 2017 (FCDL'17), 8 pages.

https://doi.org/10.1145/nnnnnnn.nnnnnnn

\section{INTRODUCTION}

Scientific articles are among the most valuable textual records of human knowledge, but the growth rate of the volume of academic publications, which some authors estimate double every nine years, [5], makes it impossible for researchers, reviewers and research

Permission to make digital or hard copies of all or part of this work for personal or classroom use is granted without fee provided that copies are not made or distributed for profit or commercial advantage and that copies bear this notice and the full citation on the first page. Copyrights for components of this work owned by others than ACM must be honored. Abstracting with credit is permitted. To copy otherwise, or republish, to post on servers or to redistribute to lists, requires prior specific permission and/or a

fee. Request permissions from permissions@acm.org.

FCDL'17, June 2017, Toronto, Canada

(c) 2017 Association for Computing Machinery.

ACM ISBN 978-x-xxxx-xxxx-x/YY/MM...\$15.00

https://doi.org/10.1145/nnnnnnn.nnnnnnn policy makers to keep up with recent advances-even within welldelimited fields. This fact calls for new ideas and applications that contribute to much-needed solutions for this potential bottleneck in scientific progress, opening interesting research avenues-in particular, in the areas of Natural Language Processing (NLP), Information Retrieval (IR) and Data Visualization.

In this work we present research contributing to an on-line system that enables the exploration of scientific knowledge disseminated through research papers. Specific tools are adapted and/or developed and integrated in order to assess their potential for the interactive navigation of collections of papers at different granularity levels: from the in-depth exploration of a single-document to the analysis of dynamically defined subsets of the corpus on which the user can zoom in. By incorporating the temporal dimension into the analyses, it is also possible to study the evolution of metadata-based information (e.g. participation of authors and institutions in different editions of a conference or journal), as well as of data extracted by linguistic analyses (e.g. the rise or decline of research topics in the context of a conference throughout the years) or combinations of both (e.g. changes in the prevalence of research topics in particular geographic regions).

As a case study we retrieved, processed and made available online knowledge contained in papers presented at the Spanish Society for Natural Language Processing (SEPLN) conference between 2008 and 2016 and, to illustrate potential use scenarios of the described tools, we briefly analyzed the evolution of topics automatically extracted from the papers presented at the conference in this period.

The contributions of this paper include:

(1) The development of a multilingual (English, Spanish) tool for deep analysis of scientific articles;

(2) The development of an on-line system for semantic exploration of large collections of full scientific articles;

(3) A case study in the NLP area to demonstrate the added value of our approach.

The rest of this paper is organized as follows: in Section 2 we describe tools and methods used to extract, analyze and index metadata and linguistic knowledge obtained from PDF files of scientific articles. Section 3 describes on-line resources created and made available for accessing a collection of papers and visualizing the data extracted from it. In Section 4 we show an example of an analysis of the evolution of semantic topics in the corpus for which we rely on the resources described in this paper. Section 5 reports 
related work in the areas of analysis and visualization of collections of scientific texts and we conclude by describing areas where we see potential for extending and improving our current results.

\section{EXTRACTING, ANALYZING AND INDEXING SCIENTIFIC KNOWLEDGE}

In this section we first-in subsection 2.1-describe the corpus developed for this work and how it was collected. Next, in subsection 2.2 , the PDF extraction component of the system is introduced and the results of its evaluation are discussed. In 2.3 we explained the implemented mechanism in order to unambiguously associate authors their affiliations and geographic locations to the analyzed papers, as well as preliminary results of evaluating it. Subsection 2.4 describes the linguistic processes applied in order to enrich the extracted data with with semantic and discourse-level information and, finally, subsection 2.5 described the tools used in order to index and query the data resulting of the mining processes.

\subsection{The SEPLN corpus}

The SEPLN is a non-profit organization created in 1983 focused on the promotion of research in the area of NLP, which has, among its main activities, the organization of an yearly international conference. The papers presented at the SEPLN conference are published in the peer-reviewed journal "Procesamiento del Lenguaje Natural" (two issues per year) and made openly available on-line for the benefit of the NLP research community. ${ }^{1}$

The SEPLN accepts and publishes papers written both in Spanish and English. Since 2008 the format of the SEPLN papers has been standardized, including titles, abstracts, and keywords both in English and Spanish, which makes this collection particularly interesting as a source for multilingual text analyses.

Working with the subset of SEPLN papers in the period 20082016 allows us to apply a set of previously developed tools targeted at English texts while, at the same time, opens up the possibility of adapting these tools in order to extend the analyses to papers written in Spanish, which we have partially done in the context of this project.

To build the corpus for our case study we collected 505 PDF documents corresponding to the articles published in the SEPLN Journal volumes 40 (2008) to 57 (2016), which includes the following subsets of documents: 374 articles (of about 8 pages), 47 demo articles (2-4 pages), 50 projects' descriptions (about 2-4 pages), 33 $\mathrm{PhD}$ thesis' descriptions (about 2-4 pages), and 1 book review (2 pages). The corpus has a proportion of papers per language of about $54 \%$ for Spanish and $46 \%$ for English.

\subsection{Information extraction from PDF files}

The first processing step is the extraction of the textual informationmetadata and papers' contents-from the PDF versions openly available at the SEPLN Journal website.

Considering that our final goal is to make scientific articles explorable in a user-friendly way through our web platform, it was important for us to not only retrieve a paper's metadata and textual contents but to also extract information about its structure and

\footnotetext{
${ }^{1}$ http://journal.sepln.org/sepln/ojs/ojs/index.php/pln
}

format in order to expose its contents in a way that maintains the original layout and preserves essential information such as that contained in tables, footnotes, figures and graphs. Information about the documents' structural elements, as well as non-textual information contained in the papers, is generally lost in the PDF-to-text extraction processes implemented by state-of-the-art tools such as GROBID ${ }^{2}{ }^{2}$ CERMINE, ${ }^{3}$ and PDFX. ${ }^{4}$

We developed, as part of this project, a layout-preserving PDF extractor that supports the most frequent formats of scientific papersincluding the one used by the SEPLN journal-and can handle textual contents in multiple languages (currently supporting English and Spanish). The output of the PDF extraction phase is a pair of XML and HTML documents containing information about structural elements of the paper and its original layout, respectively. A mapping between the structural elements in XML and their layout in HTML (by means of DIV elements) is produced, which is then exploited by the single-document visualization of the paper to properly highlight specific portions of its content.

The PDF content extraction algorithm is able to extract the following structural elements of scientific articles: title, second title (in case it exists), authors' names, affiliation and email, abstract(s), categories, keyword(s), sections title and textual content, acknowledgements, bibliographic entries, figure and table captions, among others.

The algorithm has five sequential phases: 1) PDF-to-HTML conversion; 2) computation of document-level statistics of HTML/CSS elements; 3) rule-based content detection and extraction; 4) language prediction and; 5) XML generation and validation.

The rules for the extraction of the papers' structural elements are based on: 1) finite states that capture the papers' structure (e.g. abstracts come after titles); 2) HTML attributes statistics (e.g. the most used textual font in the paper and its size); 3) CSS properties' values (e.g. values indicating the $y$-Axis position of an element in the page.), 4) regular expressions covering one or several languages (e.g. detecting strings such as 'Abstract:' or 'Resumen:' to identify the abstract), 5) HTML local element specific data (e.g. distance between the current and previous HTML tag elements), and 6) manuallytuned offset thresholds (e.g. the maximum distance between the title and a line of text below the title to be considered part of the title).

The PDF extractor uses the following external software libraries: 1) pdf2htmlEX ${ }^{5}$ to extract an HTML version of the original PDF, 2) JSoup ${ }^{6}$ to parse the HTML content and generate the XML output, 3) optimaize ${ }^{7}$ Java API to identify the language of each structural element extracted, and 4) $\mathrm{JTidy}^{8}$ to perform XML validation.

We evaluated the quality of the extraction of structured textual contents from PDF files by creating a gold standard set of 30 SEPLN articles that were manually annotated in order to spot: title(s), abstract(s), list of keywords, section headers (up to a depth of three levels), paragraphs, table captions, figure captions and bibliographic

\footnotetext{
${ }^{2}$ https://github.com/kermitt2/grobid

${ }^{3}$ http://cermine.ceon.pl/

${ }^{4}$ http://pdfx.cs.man.ac.uk/

${ }^{5}$ https://github.com/coolwanglu/pdf2htmlEX

${ }^{6}$ https://jsoup.org/

${ }^{7}$ https://github.com/optimaize/language-detector

${ }^{8}$ http://jtidy.sourceforge.net/
} 
Table 1: Evaluation of the extraction of structural elements

\begin{tabular}{llll}
\hline Class & Prec. & Recall & F1 \\
\hline Title & 1.000 & 1.000 & 1.000 \\
Abstract & 0.921 & 0.928 & 0.890 \\
Keywords & 0.963 & 0.917 & 0.915 \\
H1 -section title- & 0.978 & 0.807 & 0.876 \\
H2 -subsection title- & 0.875 & 0.876 & 0.864 \\
H3 -subsubsection title- & 0.875 & 1.000 & 0.917 \\
Paragraph & 0.994 & 0.874 & 0.923 \\
Table caption & 0.549 & 0.879 & 0.664 \\
Figure caption & 0.584 & 0.922 & 0.691 \\
BibEntry & 0.923 & 0.915 & 0.919 \\
\hline Avg. weighted & & & \\
by class freq. & 0.878 & 0.976 & 0.917
\end{tabular}

entries. The result of this evaluation are shown in Table 1 . We obtain an average F1 score of 0.917 .

Most of the PDF-to-XML extraction errors are due to: 1) papers without valid textual content extracted from the original PDF file and 2) papers that do not follow the logical structure of a regular paper (e.g. papers without abstract, papers without section titles, etc.).

In addition to the spotting of structural elements of the papers, the PDF extractor identifies the authors' names, e-mails and portions of text in the article that correspond to information about the authors' affiliations, which is then used to identify research institutions and to geographically locate them, as described in the next section.

\subsection{Identifying authors, affiliations and locations}

Knowing the affiliation of the papers' authors at the time of their writing is essential if we intend to analyze, for instance, institutional contributions to particular research topics-as it is to integrate geographical dimensions to the study of the evolution of a scientific discipline.

The availability of this information is, nevertheless, very limited. Online services such as Google Scholar ${ }^{9}$ only display information about the current affiliation of authors and, according to their terms of service, it not possible to use it with a method other than the interface and the instructions that the company provides. ${ }^{10}$

The DBLP service, ${ }^{11}$ a joint initiative of the University of Trier and Schloss Dagstuhl, offers openly available metadata about papers in the area of computer science. Bibliographic entries are available in DBLP for all SEPLN papers considered in our study, including the paper's title, year of publication and authors, who are uniquely identified by their DBLP URI. This is a valuable source of information that we exploit in our system, as described below. But in terms of author affiliations, the information available in DBLP has two limitations: as in the case of Google Scholar, the association between authors and institutions is offered only for the most recent

\footnotetext{
${ }^{9}$ https://scholar.google.com/

${ }^{10} \mathrm{https} / / / \mathrm{www} . g o o g l e . c o m /$ intl/en/policies/terms/

${ }^{11} \mathrm{http}: / /$ dblp2.uni-trier.de/
}

affiliation and it is only available for a somewhat small percentage of the authors. Considering all the numbers of the SEPLN Journal, only $13 \%$ of the authors (169 out of 1261 ) have affiliation information available on DBLP.

Microsoft Academic ${ }^{12}$ - probably the most comprehensive source of exploitable bibliographic information for scientific articles-is available as a web search service and through a REST API. It includes some information about author affiliations for particular papers, although limited. Considering full collection of SEPLN Journal papers, only $6.7 \%$ (323 out of 4837) author-paper instances have author affiliation information in Microsoft Academic. Moreover, it presents some errors that make it unsuitable for the analysis of the SEPLN collection, in particular. For instance, authors affiliated to the University of Alicante, in Spain, or the Computing Research Center (Centro de Investigación en Computación) in Mexico, are incorrectly located by Microsoft Academic in the United States and assigned United Airlines and the Council for Independent Colleges, respectively, as their affiliations. Presumably because their respective acronyms match.

In order to overcome this problem we implemented a process that, based on the papers' DBLP entries and the emails and affiliation texts obtained by our PDF extractor, attempts to uniquely identify the main institution to which each author is affiliated in each paper. This process makes use of the IP Geolocation ${ }^{13}$, DBpedia Spotlight ${ }^{14}$, Babelfy ${ }^{15}$ and Google Maps ${ }^{16}$ web services in order to identify candidate affiliations, determine if they correspond to research institutions and find their geographical information (including city, country and coordinates). If an institution cannot be identified based on the data extracted from the PDF file and there is affiliation information available for the author in DBLP, it is used as default. If the institution is found in DBpedia its URI is used as the institution unique identifier. In other cases, a new URI is assigned to it. We plan to expose this information in a future work as Linked Open Data, which we believe would be a valuable contribution for the study of academic bibliographies.

We evaluated this method of assignment of affiliations to authors by randomly generating a $10 \%$ subset of the 1,585 author-paper instances available in the corpus and manually determining, for each one: 1) if a unique email address is associated with an author by the PDF extractor and it does correspond to the correct author's email, 2) if the organization assigned to the author corresponds to the correct author's affiliation in the paper, 3) if the organization is uniquely identified both by its name in the preferred language and by its URI, and 4) if the country and city where the institution is located are in fact those identified.

The precision values obtained are shown in Table 2. Using the authors' emails in order to identify their affiliation we obtain a precision of 0.83 for the unique identification of the institutions.

In our current implementation, unique e-mails are extracted from the PDF files and linked to the right author in $75 \%$ of the cases. We are now working towards extending the coverage of our PDF extractor to less frequent formats which will, in turn, improve the

\footnotetext{
${ }^{12}$ https://academic.microsoft.com/

${ }^{13} \mathrm{http}: / /$ ip-api.com/

${ }^{14} \mathrm{http}: / /$ demo.dbpedia-spotlight.org/

${ }^{15} \mathrm{http}: / /$ babelfy.org/

${ }^{16} \mathrm{https} / /$ developers.google.com/maps/web-services/
} 
Table 2: Precision values for affiliation identification

\begin{tabular}{lcccc}
\hline Method & Affil. name & Affil. URI & City & Country \\
\hline E-Mail+Text & 0.87 & 0.83 & 0.79 & 0.91 \\
\hline
\end{tabular}

unique identification of the organizations and their geographical locations.

\subsection{Linguistic knowledge mining}

2.4.1 Textual analysis of the papers' contents. The text extracted from the PDF file of each paper feeds a linguistic processing pipeline, built by relying on and extending [16], that produces a set of annotations at the sentence level, which allows for deeper exploration of the papers' contents. In particular, each sentence is assigned a rhetorical class that conveys information about the sentence's purpose in the text, contributing to characterize the discursive structure of the article. As described in Section 3, this information is then exploited by the search and visualization modules of the platform, allowing the user to explore, for instance, the evolution of a topic in a research community as reflected by its use in different rhetorical contexts.

The set of rhetorical functions considered includes the following five classes: Approach, Background, Challenge, Outcome and Future work. Sentences for which a specific rhetorical category cannot be determined are annotated as Unspecified.

A set of features is produced as a result of the linguistic processing pipeline, which includes sentence splitting, tokenization, POS-tagging and dependency parsing of the paper's contents. These features are used by a logistic regression classifier-trained over the DrInventor Multi-layer Scientific Corpus [10]-in order to assign a rhetorical class to each sentence.

Information about the language of the text returned by the PDF extractor is used in order to decide which set of tools to use in each case. We rely on openly available NLP tools for these tasks. In particular, we make use of the sentence splitter and tokenizer plugins integrated into the GATE framework [7], adapted for the specificities of the scientific articles, and of the POS-tagger and dependency parser available as part of the MATE modules for natural language analysis [4]. ${ }^{17}$

In addition to their rhetorical class, the papers' sentences are automatically annotated with relevance scores that reflect their suitability to be part of an extractive summary of the paper. Several scores are calculated based on text summarization algorithms including: term-frequency and centroid [17, 18], LexRank [9] and TextRank [13]. These relevance scores are available in order to provide sentence highlights in a single document visualization interface.

2.4.2 Identifying the topics addressed by the papers. We use three sources of information in order to analyze the evolution of research topics in the context of a particular conference: 1) the keywords assigned by the paper's authors, which are extracted in the PDF processing phase for all the available languages, 2) Babelfy [14], which is used to spot mentions of concepts and named entities that occur in each paper associating a specific sense (synsets) to

\footnotetext{
${ }^{17}$ https://code.google.com/p/mate-tools/
}

each one ${ }^{18}$ and 3) topics obtained by applying the latent Dirichlet allocation (LDA) [3] topic modeling method, as described below.

2.4.3 Topic modeling. Analyzing the results obtained by running LDA topic modeling with 10, 20, 30 and 50 topics for each language, we observed that the most semantically consistent results were obtained by setting a number between 20 and 30 topics. We therefore processed the articles in our collection obtaining, for each one, a vector that contains the paper's probability distribution over 30 topics. The 20 most clearly defined topics were then manually picked to be used in the visualizations.

Table 3 shows this set of 20 topics together with the top 10 words associated to each one in the corpus and the labels we assigned to identify the topic in the visualizations.

It is relevant to note that the topics described here are those obtained by texts available in English, including abstracts in English of papers written in Spanish, which could introduce some bias in the results. ${ }^{19}$ We plan to address this limitation in future versions of the tools in order to work with a unified set of topics obtained from texts in both languages.

2.4.4 Papers and authors similarity. Based on the resulting topic model we computed the authors' topics as the mean of their respective papers' vectors. We then assigned, to each paper and author, their 30 most similar ${ }^{20}$ papers and authors, respectively, based on their topics distributions, which will be used to include papers and authors recommendations based on topic similarity.

\subsection{Data indexing}

The data extracted in the previously described processes is indexed so as to be explored through custom searches and visualizations. We use Elasticsearch ${ }^{21}$ as the search engine solution for the project. Based on Apache Lucene, ${ }^{22}$ its robustness and efficiency for the execution of real-time searches are valuable assets of Elasticsearch, as it is its support of faceted searches-through the aggregations framework-, which makes it possible to gradually explore the dataset. By relying on a schema-less data storage solution it is possible to enrich the indexed data incrementally.

The availability of Elasticsearch clients for the most popular programming languages provides a great flexibility in terms of both indexing and accessing the data. In particular, we use extensively the elasticsearch.js JavaScript API ${ }^{23}$ to search and retrieve the data displayed on our web platform and the Java client ${ }^{24}$ in order to index the analyzed papers' contents.

A determining feature for the election of Elasticsearch in our project is its integration with Kibana, ${ }^{25}$ a data visualization application bundled with the search engine on which we base several of the charts implemented in our platform.

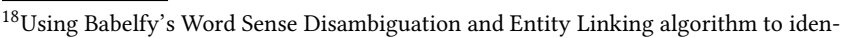
tify occurrences of language-independent synsets is particularly useful to study the evolution of concepts in a multilingual corpus such as ours.

${ }^{19} \mathrm{An}$ analoguous analysis was made in order to extract topics from the texts in Spanish.

${ }^{20}$ We used cosine distance between their respective topic vectors as a measure of the similarity between each pair of papers/authors.

${ }^{21} \mathrm{https}$ ://www.elastic.co/

${ }^{22}$ https://lucene.apache.org/

${ }^{23} \mathrm{http} / / /$ www.elastic.co/guide/en/elasticsearch/client/javascript-api/current/

${ }^{24}$ https://www.elastic.co/guide/en/elasticsearch/client/java-api/current/client.html

${ }^{25}$ https://www.elastic.co/products/kibana
} 
Table 3: Topics

\begin{tabular}{|c|c|c|c|c|c|c|c|c|c|c|}
\hline \multirow{2}{*}{$\frac{\text { Topic label }}{\text { speech systems }}$} & \multicolumn{10}{|c|}{ Top-10 words } \\
\hline & speech & system & systems & basque & module & spanish & audio & segments & language & speaker \\
\hline social media & tweets & twitter & sentiment & tweet & users & user & social & media & analysis & spanish \\
\hline clustering & clustering & cluster & documents & clusters & document & values & algorithm & measure & corpora & consensus \\
\hline biomedical text mining & medical & & clinical & negation & concept & biomedical & concepts & extraction & information & entities \\
\hline paraphrasing & paraphrase & paraphrases & noun & paraphrasing & pairs & subject & object & compound & pair & entailment \\
\hline knowledge resources & semantic & lexical & relations & model & ontology & knowledge & concepts & meaning & conceptual & events \\
\hline text simplification & simplification & text & sentences & sentence & corpus & texts & original & simplified & subjects & zero \\
\hline machine translation & translation & language & model & alignment & sentences & source & sentence & words & parallel & system \\
\hline opinion mining & reviews & polarity & opinion & aspect & words & features & opinions & product & word & feature \\
\hline coreference resolution & mentions & coreference & mention & resolution & entity & system & discourse & muc & precision & relation \\
\hline sentiment analysis & emotion & polarity & text & emotions & sentiment & classification & emotional & level & negative & positive \\
\hline annotation & annotation & corpus & should & interaction & knowledge & analysis & tool & annotations & will & intention \\
\hline dependency parsing & dependency & parsing & parsers & training & parser & sentences & nivre & sentence & feature & las \\
\hline topic analysis & document & documents & plagiarism & topic & topics & detection & $\mathrm{mfs}$ & reference & text & time \\
\hline anaphora resolution & anaphora & patterns & personality & relation & antecedent & spam & hypernym & referring & pattern & cases \\
\hline pos tagging & pos & word & tags & corpus & $\operatorname{tag}$ & tagger & words & tagset & spanish & tokens \\
\hline resources and tools & project & resources & will & information & language & tools & data & languages & processing & available \\
\hline word sense disambiguation & word & sense & wordnet & semantic & senses & wsd & disambiguation & knowledge & method & words \\
\hline dialogue systems & user & dialogue & system & information & models & model & items & speech & goals & interaction \\
\hline web search & web & pages & information & query & page & search & documents & retrieval & results & tags \\
\hline
\end{tabular}

\section{MAKING SCIENTIFIC KNOWLEDGE AVAILABLE: ON-LINE PUBLICATION AND VISUALIZATION}

The data resulting from mining the content of the SEPLN articles can be accessed through a web platform available at http: //scipub-taln.upf.edu/sepln, where the users can explore the collection by performing full text queries or by browsing it with the help of a number of exposed filters that are dynamically populated from Elasticsearch in order to support faceted searches. These filters include: keywords, Babelnet synsets, ${ }^{26}$ authors, affiliations, countries and cities. The displayed papers can also be limited to those published within a range of years.

\subsection{Visualizations}

The web platform allows the users to explore the papers' mined contents from the perspective of the whole collection or at the individual level-by a single-document visualization that exploits the mappings between structural and layout elements obtained in the PDF extraction phase. This visualization allows the user to analyze the discursive structure of the paper by rapidly identifying the distribution of the rhetorical categories in it, and to obtain an extractive summary of its contents by highlighting those sentences annotated according to the results of the summarization algorithms described in Section 2.

At the corpus level, the following visualizations are available:

(1) Date histogram of published papers

(2) Heatmap of keywords used throughout the years

(3) Word cloud of keywords

(4) Evolution of Babelnet synsets frequency through rhetorical categories

(5) Date histogram of topics

(6) Force-directed graphs of papers/authors and topics

\footnotetext{
${ }^{26}$ In the case of the Babelnet synsets, the most frequent lexicalization in the corpus is used as filter value. Filtering the results by the synset lexicalized by the term 'parser' in English would also retrieve documents where the term 'syntactic analysis' occur, as well as their variations in Spanish.
}

(7) Geo-spatial visualization of publications and collaborations

The visualizations are dynamically generated based on the user's queries and applied filters, making it possible to restrict the data to explore based, for example, on the papers' authors, their affiliations or geographic locations.

Figure 1: Graph of papers filtered by machine learning

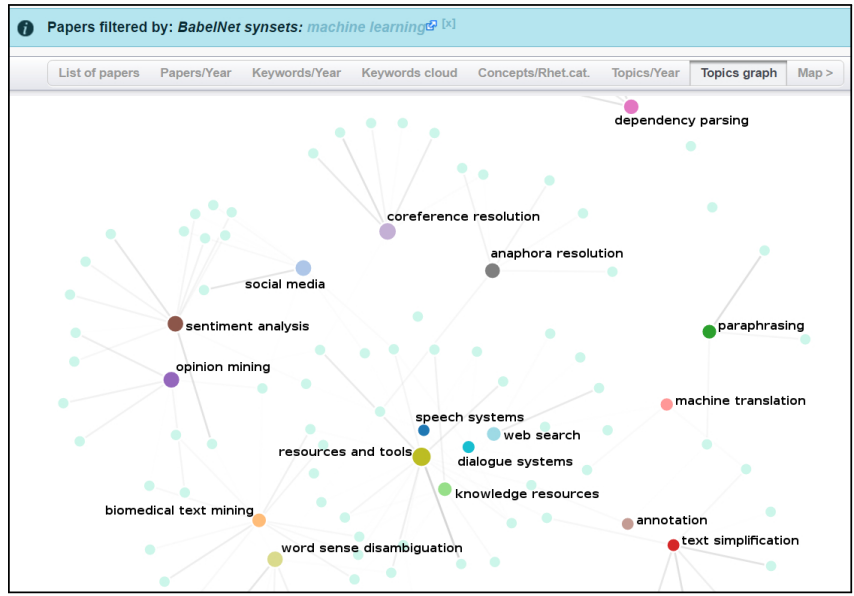

3.1.1 Geo-referenced visualization of publications and collaborations. Choropleth maps are generated based on the authors' affiliations and their geographic localizations, identified by the process described in Section 2. This makes it possible to explore collaboration links between research institutions in different countries or regions, as well as to analyze the process of internationalization of a venue-such as the SEPLN annual conference.

These visualizations are based on scalable vector representations of the maps enriched with data and event-based responsiveness provided by the D3.js library [6]. In particular, we use the D3-based 
library DataMaps. ${ }^{27}$ The geographical elements-a world map with country borders and map of Spain with Spanish provinces-are represented in the TopofSON format, ${ }^{28}$ an implementation of the GeofSON IETF specification. ${ }^{29}$

The geo-visualizations in our platform are conceived as interactive devices that the user can take advantage of in order to dynamically explore the data. By clicking on two countries, for instance, it is possible to promptly find the number of collaborations between institutions located in them. By selecting a particular region (country or province), a text area is displayed on top of the map with detailed information about the number of publications with the involvement of research institutions in it. This textual information contains links that can, in turn, be used to apply filters and further restrict the displayed results.

3.1.2 Topic visualizations. The current web platform offers the possibility of visualizing the distribution of topics in the SEPLN papers-or a user-defined subset of them-over a period of time: a date histogram graphic shows the evolution of each topic based on the average probability of being associated to the subset of the papers published each year. This visualization is implemented with one of the chart creation tools available in Kibana.

An alternative view of the topics distribution for papers and authors is available by means of a force-directed graph visualization [11], where the nodes represent papers and topics and the links indicate the probability of occurrence of a topic within a paper. Each topic is represented by a node of a different color. While the size of the paper nodes is the same for all the papers, in the case of the topic nodes we wanted their size to reflect their relevance in the selected set of documents. Therefore-for topics in which the sum of their probabilities in the set of documents is greater than $e$-the radius of their nodes is proportional to the logarithm of their total weight, as described by formula (1), where $s$ is the minimum radius for the topic nodes and $C$ refers to the filtered set of papers. In the current implementation, $s$ is equal to 5 pixels.

$$
r_{t}=\max \left\{s, s \times \log \left(\sum_{p \in C} w_{t p}\right)\right\}
$$

The weight ${ }^{30}$ of a topic in a paper, $w_{t p}$, is represented in the graph by the shade of the link between them: topics that have a greater weight give origin to darker lines between paper and topic nodes, while topics too weakly associated with a paperwith a weight below a pre-defined threshold-are omitted from the visualization. Similarly, only topics for which the sum of their weights in the set of displayed papers is above the threshold (i.e. there is at least one visible link between a paper and the topic) are displayed as graph nodes.

Figure 1 partially shows the graph obtained by filtering the SEPLN papers by the Babelnet synset corresponding to the term machine learning.

In the same way that the users can visualize linkages between papers and topics by means of the force-directed graphs, they can

\footnotetext{
$\overline{{ }^{27} \text { http://datamaps.github.io/ }}$

${ }^{28} \mathrm{https}$ //github.com/topojson/topojson

${ }^{29} \mathrm{http}: / /$ geojson.org/

${ }^{30}$ We use the term weight here to refer to the probability of the participation of a topic in the topic mix of the document.
}

choose instead to display the papers' authors as nodes in the graph. In this case, the links between the authors and the topics are generated based on the average participation of the topic in the author's papers, as described in Section 2.

The force-graphs were developed with the D3.js library. They are based on Mike Bostock's original example, ${ }^{31}$ and incorporate some additional features based on developments by Simon Raper, ${ }^{32}$ including the possibility of exploring a particular region of the graph in more detail, which is particularly useful when visualizing a large set of papers.

By clicking on a paper's node, a text box is displayed on the top of the visualization with the paper's title, publication year and authors. The title includes a link that can be followed in order to switch to the list view of the paper and from there, to access its detailed information and, potentially, its contents by means of the single-document visualization. If the node clicked represents a topic, instead, a list with links to all the papers/authors related with the corresponding topic is displayed.

\section{USAGE EXAMPLE}

If we look at the force graph built from the topic distributions we can see that the nodes of the topics corresponding to the concepts opinion mining and sentiment analysis are close to each other. As an example of an analysis based on the tools described in this paper we explore, in this section, how these two concepts are related in the papers included in the SEPLN corpus.

We first obtain the lists of papers most closely related with each of the topics by selecting the corresponding nodes in the graph. The top-10 papers obtained in each case, with their weights, are shown in Tables 4 and 5.

From the lists obtained we can see that there are no common papers among the ten most strongly related with the two topics. ${ }^{33}$ So, even if the two topics are connected by some paper nodes in the graph, which means that there are enough articles in the corpus with a weight above a certain threshold to keep them close together, the terms that are most probably related with each topic differ in general in the collection (which explains why the two topics are generated, in the first place).

It is interesting to take a look at the chart that shows the evolution of the topics throughout the period covered by the corpus (Figure 2), where it can be seen how the terms related to these two topics are used in complementary ways.

In particular, if we focus on the period 2012-2016, when the two topics are more prominent, a hypothesis could be formulated in relation to a potential terminological shift, in 2014, from the terms related to the topic sentiment analysis to those related to opinion mining.

By considering the evolution of the also closely related topic social media in the period, it can be observed that this research area has captured constant increasing interest in the NLP community since 2011 and until 2015. In an initial period, research around social networking platforms was mainly focused on the study of the polarity of the messages, which is consistent with the observed

\footnotetext{
${ }^{31}$ https://bl.ocks.org/mbostock/4062045

${ }^{32} \mathrm{http}$ ///www.coppelia.io/2014/07/an-a-to-z-of-extra-features-for-the-d3-force-layout/

${ }^{33}$ Analyzing the texts of these papers we can confirm that the topics distribution correctly reflects the papers contents, even if some of the titles might suggest otherwise.
} 
Table 4: Top-10 papers with topic sentiment analysis

\begin{tabular}{lll}
\hline Title & Year & $\begin{array}{l}\text { Topic } \\
\text { weight }\end{array}$ \\
\hline $\begin{array}{l}\text { Applying a culture dependent emotion trig- } \\
\text { gers database for text valence and emotion } \\
\text { classification }\end{array}$ & 0.57 \\
$\begin{array}{l}\text { SINAI en TASS 2012 } \\
\text { A social tag-based dimensional model }\end{array}$ & 2013 & 0.53 \\
$\begin{array}{l}\text { of emotions: Building cross-domain folk- } \\
\text { sonomies }\end{array}$ & 0.52 \\
$\begin{array}{l}\text { TASS 2015 - The Evolution of the Spanish } \\
\begin{array}{l}\text { Opinion Mining Systems } \\
\text { Automatic prediction of emotions from text }\end{array}\end{array}$ & 2016 \\
$\begin{array}{l}\text { in Spanish for expressive speech synthesis } \\
\text { in the chat domain }\end{array}$ & 0.38 \\
$\begin{array}{l}\text { TASS - Workshop on Sentiment Analysis at } \\
\text { SEPLN }\end{array}$ & 2013 & 0.36 \\
$\begin{array}{l}\text { Lexicon-Based Sentiment Analysis of Twit- } \\
\text { ter Messages in Spanish }\end{array}$ & 2013 & 0.36 \\
$\begin{array}{l}\text { TASS 2014 - The Challenge of Aspect-based } \\
\begin{array}{l}\text { Sentiment Analysis } \\
\text { Análisis de Valoraciones de Usuario de Hote- }\end{array}\end{array}$ & 2015 & 0.35 \\
$\begin{array}{l}\text { les con Sentitext: un sistema de análisis de } \\
\text { sentimiento independiente del dominio }\end{array}$ & & \\
$\begin{array}{l}\text { TASS 2013 - A Second Step in Reputation } \\
\text { Analysis in Spanish }\end{array}$ & 2014 & 0.34 \\
\hline
\end{tabular}

raise of the topic sentiment analysis until 2013. In later years, other areas of textual on-line communication through social networks have captured the interest of the research community, including the exploration of changes in users' habits and preferences, such as the analysis of political orientation or churn detection through the study of social media posts. The research area seemed to have turned, therefore, to more general and varied analyses that can be characterized as opinion-related research, which could also explain, in part, the more recent raise of the term opinion mining.

Further analyses of the corpus might provide other elements that could contribute to explain the observed evolution of these topics. Finer-grained analyses could consider, for instance, the impact of the TASS Workshop on Sentiment Analysis in the context of the SEPLN conference, ${ }^{34}$ whose first edition was held in 2012 and which could be an additional explanation for the increased usage of social media-related terminology by the SEPLN community since that year. Although this would not explain, by itself, the decline of the topic sentiment analysis in favor of opinion mining after that year.

Another partial explanation could be formulated in relation to a potential bias introduced in our analysis by the fact that papers written in English and Spanish have different contributions to the definition of the LDA topics, as explained in Section 2. But this does not seem determining in explaining the raise of the topic opinion mining since 2014, because the observed variations in the period are similar if we exclude from the analysis the papers written in Spanish. Also, the total contributions of the articles written in Spanish to

\footnotetext{
${ }^{34}$ http://www.sepln.org/workshops/tass/
}

Table 5: Top-10 papers with topic opinion mining

\begin{tabular}{lll}
\hline Title & Year & $\begin{array}{l}\text { Topic } \\
\text { weight }\end{array}$ \\
\hline $\begin{array}{l}\text { Función de las secuencias narrativas en la } \\
\text { clasificación de la polaridad de reviews }\end{array}$ & 2014 & 0.48 \\
$\begin{array}{l}\text { Learning a Statistical Model of Product As- } \\
\text { pects for Sentiment Analysis }\end{array}$ & 2012 & 0.47 \\
$\begin{array}{l}\text { A Semantic Relatedness Approach to Classi- } \\
\text { fying Opinion from Web Reviews }\end{array}$ & 2009 & 0.45 \\
$\begin{array}{l}\text { Enriching User Reviews Through An Opin- } \\
\text { ion Extraction System }\end{array}$ & 2015 & 0.41 \\
$\begin{array}{l}\text { Polarity analysis of reviews based on the } \\
\text { omission of asymmetric sentences }\end{array}$ & 2015 & 0.40 \\
$\begin{array}{l}\text { Unsupervised acquisition of domain aspect } \\
\text { terms for Aspect Based Opinion Mining }\end{array}$ & 2014 & 0.39 \\
$\begin{array}{l}\text { Unsupervised Word Polarity Tagging by Ex- } \\
\text { ploiting Continuous Word Representations }\end{array}$ & 2015 & 0.34 \\
$\begin{array}{l}\text { eSOLHotel: Generación de un lexicón de } \\
\text { opinión en español adaptado al dominio } \\
\text { turístico }\end{array}$ & 2015 & 0.29 \\
$\begin{array}{l}\text { Inducción de un Lexicón de Opinión Orien- } \\
\text { tado al Dominio }\end{array}$ & 2009 & 0.25 \\
$\begin{array}{l}\text { Is this Tweet Satirical? A Computational Ap- } \\
\text { proach for Satire Detection in Spanish }\end{array}$ & 2015 & 0.24 \\
\hline
\end{tabular}

Figure 2: Evolution of sample topics

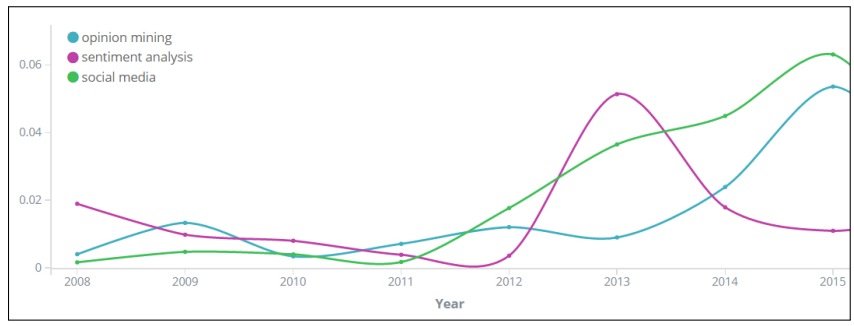

both topics are comparable. If we consider all the articles retrieved for each topic in the graph visualization we observe that the average contribution of the Spanish documents is of 0.16 and 0.18 to the topics sentiment analysis and opinion mining, respectively.

In any case, it is not our intention to exhaust the analysis of the evolution of these specific topics in the context of this article, but to point out the possibilities enabled by the tools described in this work and, in particular, their potential support to the formulation of new research questions.

\section{RELATED WORK}

Several initiatives aimed at analyzing different aspects of scientific articles have been developed around the ACL Anthology 35 [2], a corpus of more than 40,000 papers in the areas of computational linguistics and NLP from the Association for Computational Linguistics conferences and journals.

\footnotetext{
${ }^{35} \mathrm{http} / /$ aclanthology.info/
} 
The ACL Anthology Network (ANN) ${ }^{36}$ [15], maintained at the Computational Linguistics And Information Retrieval (CLAIR) group at the University of Michigan, provides an on-line interface from where more than 28,000 papers of the ACL Anthology can be accessed. The ANN functionalities includes the possibility of searching by title, contents, institution and authors, as well as browsing the collection by venue, authors and topics. It is also possible to explore a paper's statistics, its citations network, to obtain a citationbased summary of a paper's contents and to access similar papers based on a title-similarity score. The ACL Anthology Searchbench $[19]^{37}$ makes available on-line the results of the linguistic analysis of a subset of the ACL Anthology that currently includes more than 28,000 papers. Papers can be retrieved by a combination of filters populated with metadata extracted from the articles, as well as by performing semantic searches enabled by normalized semantic representations obtained as the result of an automatic tuple extraction process.

In [1] and [12] the authors use LDA modeling to perform topicbased analyses of the ACL Anthology in order to explore research trajectories of authors across subfields of computational linguistics and NLP, as well as the variation of research topic trends and terminological shifts produced in the area in specific periods of time. Antecedents in the integration of text analysis and visualization tools to study corpora of scientific articles include the Action Science Explorer (ASE) [8], which provides a series of visual tools to explore citation networks and contexts in addition to generate automatic citations-based multi-document summaries of a selected set of papers.

\section{CONCLUSIONS AND FUTURE WORK}

The availability of tools for deep analysis of scientific texts, together with flexible visualization and exploration interfaces, plays a major role in allowing the effective exploitation of knowledge contained in collections of scientific texts. Access to recent and past scientific discoveries, methods, and techniques is essential for scientific inquiry activities which include, among others: (i) finding open or solved problems, (ii) understanding research fields' dynamics or evolution, (iii) discovering experts in specific scientific areas, or (iv) understanding the advantages and limitations of current solutions. This paper presented a suite of scientific text analysis tools able to enrich research papers with metadata, semantics, and rhetorical information. The collective analysis of collections of research papers crawled from open-access respositories is provided through a newly developed interface which supports novel faceted-search functionalities to understand research collaboration and topic-evolution. All the tools are being made accessible through a software library for the benefit of the scientific community. We are currently focused on the intrinsic evaluation of various aspects of our mining tool including the comparison of our PDF extractor to state-of-the-art tools.

Due to time limitation it was not possible to conduct a user validation and evaluation of the developed tools-in particular, the search and visualization platform-which is planned as future work. The extension of some of our components-such as the rhetorical

\footnotetext{
${ }^{36} \mathrm{http}: / /$ clair.eecs.umich.edu/aan/

${ }^{37}$ http://aclasb.dfki.de/
}

classifier-to Spanish, improvement of author information extraction by relying on lexicons and external resources, and providing functionalities for citation-based summarization taking advantage of citation networks are also foreseen as follow-up paths to the work presented in this paper, as well as an analysis of the scalability of the system when dealing with larger collections.

\section{ACKNOWLEDGEMENTS}

This work is (partly) supported by the Spanish Ministry of Economy and Competitiveness under the Maria de Maeztu Units of Excellence Programme (MDM-2015-0502) and by the TUNER project (TIN201565308-C5-5-R, MINECO/FEDER, UE).

\section{REFERENCES}

[1] Ashton Anderson, Dan McFarland, and Dan Jurafsky. 2012. Towards a computational history of the acl: 1980-2008. In Proceedings of the ACL-2012 Special Workshop on Rediscovering 50 Years of Discoveries. ACL, 13-21.

[2] Steven Bird, Robert Dale, Bonnie J Dorr, Bryan R Gibson, Mark Thomas Joseph, Min-Yen Kan, Dongwon Lee, Brett Powley, Dragomir R Radev, Yee Fan Tan, and others. 2008. The ACL Anthology Reference Corpus: A Reference Dataset for Bibliographic Research in Computational Linguistics.. In LREC.

[3] David M Blei, Andrew Y Ng, and Michael I Jordan. 2003. Latent dirichlet allocation. fournal of Machine Learning Research 3, Jan (2003), 993-1022.

[4] Bernd Bohnet. 2010. Very high accuracy and fast dependency parsing is not a contradiction. In Proceedings of the 23rd International Conference on Computational Linguistics. ACL, 89-97.

[5] Lutz Bornmann and Rüdiger Mutz. 2015. Growth rates of modern science: A bibliometric analysis based on the number of publications and cited references. Journal of the Association for Information Science and Technology 66, 11 (2015), 2215-2222.

[6] Michael Bostock, Vadim Ogievetsky, and Jeffrey Heer. 2011. D ${ }^{3}$ data-driven documents. IEEE transactions on visualization and computer graphics 17, 12 (2011), 2301-2309.

[7] Hamish Cunningham, Diana Maynard, and Kalina Bontcheva. 2011. Text processing with gate. Gateway Press CA.

[8] Cody Dunne, Ben Shneiderman, Robert Gove, Judith Klavans, and Bonnie Dorr. 2012. Rapid understanding of scientific paper collections: Integrating statistics, text analytics, and visualization. Fournal of the American Society for Information Science and Technology 63, 12 (2012), 2351-2369.

[9] Günes Erkan and Dragomir R Radev. 2004. Lexrank: Graph-based lexical centrality as salience in text summarization. Journal of Artificial Intelligence Research 22 (2004), 457-479.

[10] Beatriz Fisas, Francesco Ronzano, and Horacio Saggion. 2016. A multi-layered annotated corpus of scientific papers. In Proceedings of the Tenth International Conference on Language Resources and Evaluation (LREC 2016). European Language Resources Association (ELRA), Paris, France (may 2016).

[11] Thomas MJ Fruchterman and Edward M Reingold. 1991. Graph drawing by forcedirected placement. Software: Practice and experience 21, 11 (1991), 1129-1164.

[12] David Hall, Daniel Jurafsky, and Christopher D Manning. 2008. Studying the history of ideas using topic models. In Proceedings of the conference on empirical methods in natural language processing. ACL, 363-371.

[13] R. Mihalcea and P. Tarau. 2004. TextRank: Bringing Order into Texts. In Proceedings of EMNLP-04and the 2004 Conference on Empirical Methods in NLP.

[14] Andrea Moro, Francesco Cecconi, and Roberto Navigli. 2014. Multilingual Word Sense Disambiguation and Entity Linking for Everybody. In Proceedings of the 2014 International Conference on Posters \&\#38; Demonstrations Track - Volume 1272 (ISWC-PD'14). Aachen, Germany, Germany, 25-28.

[15] Dragomir R Radev, Pradeep Muthukrishnan, and Vahed Qazvinian. 2009. The ACL anthology network corpus. In Proceedings of the 2009 Workshop on Text and Citation Analysis for Scholarly Digital Libraries. ACL, 54-61.

[16] Francesco Ronzano and Horacio Saggion. 2016. Knowledge extraction and modeling from scientific publications. In In the Proceedings of the Workshop "Semantics, Analytics, Visualisation: Enhancing Scholarly Data" co-located with the 25th International World Wide Web Conference, Montreal, Canada.

[17] Horacio Saggion. 2008. SUMMA: A robust and adaptable summarization tool. Traitement Automatique des Langues 49, 2 (2008).

[18] Horacio Saggion and Robert Gaizauskas. 2004. Multi-document summarization by cluster/profile relevance and redundancy removal. Proceedings of the Document Understanding Conference (2004), 6-7.

[19] Ulrich Schäfer, Bernd Kiefer, Christian Spurk, Jörg Steffen, and Rui Wang. 2011. The ACL anthology searchbench. In Proceedings of the 49th Annual Meeting of the ACL: Human Language Technologies: Systems Demonstrations. ACL, 7-13. 